\title{
Evidence for a projection from the globus pallidus to the entopeduncular nucleus in the rat
}

\author{
Anthony E. Kincaid ${ }^{1}$, John B. Penney Jr. ${ }^{2}$, Anne B. Young ${ }^{2}$ and Sarah W. Newman ${ }^{1}$ \\ 'Department of Anatomy and Cell Biology and 'Department of Neurology, University of Michigan, Ann Arbor, MI 48109 (U.S.A.)
}

(Received 30 January 1991; Revised version received 22 March 1991; Accepted 2 April 1991)

Keywords: Basal ganglia; Globus pallidus; Entopeduncular nucleus; Fluoro-gold; Phaseolus vulgaris-leucoagglutinin

\begin{abstract}
Iontophoretic injections of the fluorescent retrograde tract tracer, Fluoro-gold, into the entopeduncular nucleus of the rat (homologous to the internal segment of the primate globus pallidus) resulted in a substantial number of retrogradely labeled neurons in the ipsilateral globus pallidus (homologous to the external segment of the primate globus palidus). In experiments confirming this projection, iontophoretic injections of the anterograde tract tracer, Phaseolus vulgaris-leucoagglutinin, in the globus pallidus resulted in dense fiber and terminal labeling in the ipsilateral entopeduncular nucleus. This projection is topographically organized in rostral-caudal, medial-lateral and dorsal-ventral orientations.
\end{abstract}

Previous experimental research utilizing degeneration methods in primates noted the presence of a projection from the external segment of the globus pallidus to the internal segment of the globus pallidus in some reports $[8,20]$ but failed to note it in others $[1,9]$. Nauta and Mehler [10] described terminal degeneration in the primate internal globus pallidus following a lesion restricted to the external globus pallidus (case MGB 12), but stated that this may have been due to the interruption of striatofugal fibers. Recently Hazrati et al. [6] reported evidence for this projection in primate using Phaseolus vulgaris-leucoagglutinin (PHA-L) as a tract tracer. Similarly, the experimental literature on connections between the globus pallidus (GP) and the entopeduncular nucleus (EP) of the rodent provides preliminary evidence of their existence, but no description of the organization of the system. Carter and Fibiger [2] injected $\left[{ }^{3} \mathrm{H}\right]$ leucine in the rodent GP and noted the presence of grains over the EP, but were unable to determine whether these represented axons of passage or terminals. Other suggestive evidence for this projection comes from Smith and Bolam [18] and Shu and Peterson [17] who identified the presence of anterogradely labeled fibers in the EP following injections of PHA-L in the rodent GP while studying other projection targets of the GP, and from Staines [19] who reported preliminary evidence for a GP to EP projection. Physiological evidence for this

Correspondence: S.W. Newman, Department of Anatomy and Cell Biology, Room 5710 Medical Science II, University of Michigan, Ann Arbor, MI 48109-0616, U.S.A. projection has been reported by Kitai and Kita [7] who recorded antidromic activation of GP neurons with intracellular electrodes after stimulation of EP.

The objectives of this study were to investigate the existence of a projection from the GP to the EP using complementary modern neuroanatomical methods and to describe the organization of this projection.

Adult Sprague-Dawley rats (212-484 g; Harlan, Portage MI) were anesthetized with either chloral hydrate $(400 \mathrm{mg} / \mathrm{kg}$, i.p.) or ketamine/xylazine $(10 / 3 \mathrm{ratio}, 1.0$ $\mathrm{ml} / \mathrm{kg}$, i.p.) prior to placement in a stereotaxic frame. The following injection coordinates were taken from the rat atlas by Paxinos and Watson [13], for EP: AP, -2.3 to $-3.3 \mathrm{~mm}$; $\mathrm{ML},-2.5$ to $-3.2 \mathrm{~mm}$; DV, -7.4 to $-7.7 \mathrm{~mm}$ from bregma; for GP: AP, $-1.0 \mathrm{~mm}$; $\mathrm{ML}$, -3.4 to $-3.6 \mathrm{~mm}$; DV, -6.0 to $-6.5 \mathrm{~mm}$ from bregma. To eliminate the possibility of tracer leakage into the caudate-putamen ( $\mathrm{CPu}), 5$ of the GP injections were made at a $16^{\circ}$ angle from the midsagittal plane with $\mathrm{ML}$ and DV coordinates adjusted to $-1.0 \mathrm{~mm}$ and -7.0 to $-7.5 \mathrm{~mm}$ respectively, the pipette trajectory thus avoiding the $\mathrm{CPu}$.

Fluoro-gold (FG; Fluoro-chromes, Englewood, CO; $2.5 \%$ in acetic acid, $\mathrm{pH} 2.9$ ) was iontophoretically injected $(1.5-3.0 \mu \mathrm{A}, 7 \mathrm{~s}$ on $7 \mathrm{~s}$ off, for 5-16 min) into the EP of 16 adult rats via glass micropipettes with inside tip diameters of $20-45 \mu \mathrm{m}$. Following a survival time of 4-7 days the animals were perfused with $200 \mathrm{ml}$ of 0.1 $M$ sodium phosphate buffered saline (PBS) with $0.1 \%$ sodium nitrite followed by $300-400 \mathrm{ml}$ of $4 \%$ paraformaldehyde in $0.1 \mathrm{M}$ phosphate buffer (PB). The brains 
were postfixed for $1-2 \mathrm{~h}$ and cryoprotected in $20 \%$ sucrose in PB overnight. Frozen coronal or sagittal sections $(40 \mu \mathrm{m})$ were collected in $\mathrm{PB}$ with $0.1 \%$ sodium azide. The sections were mounted on gel-subbed slides, dehydrated in graded alcohols to xylene and coverslipped in DPX (Gallard-Schlesinger, Carle Place, NY).
Sections not further than $120 \mu \mathrm{m}$ apart were analyzed on either a Leitz Aristoplan or Orthoplan microscope equipped with epifluorescence. A filter block providing ultraviolet excitation was used to visualize FG labeled neurons.

PHA-L (2.5\% in PBS, pH 8.0; Vector, Burlingame,
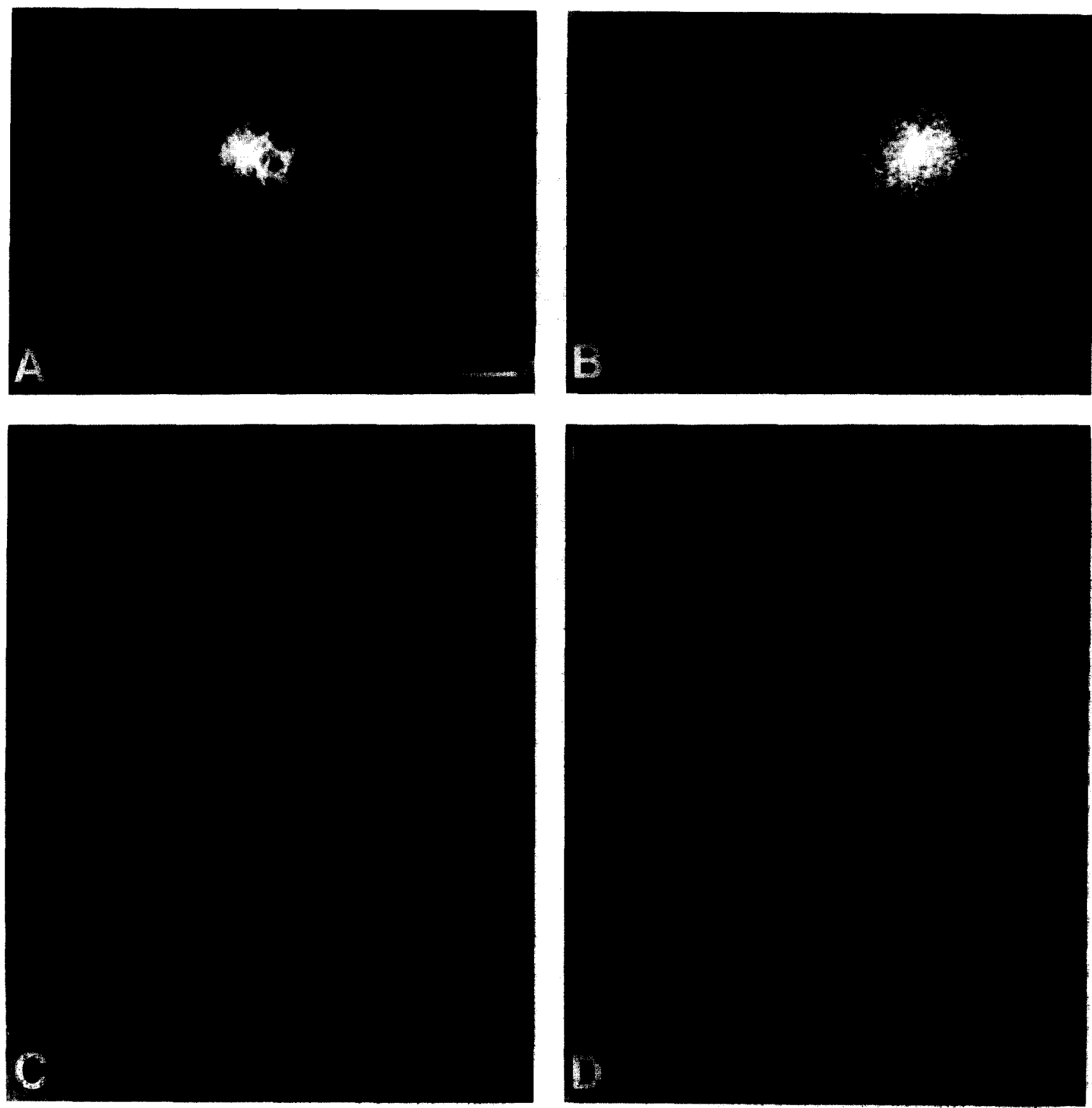

Fig. 1. FG injection sites produced retrogradely labeled neurons indicating the medial/lateral topography of projection from GP to EP. A: FG injection site centered in lateral EP with minimal spread of tracer into surrounding structures. B: FG injection site centered in medial EP with spread into the lateral hypothalamus. C: FG labeled neurons restricted to the lateral part of the ipsilateral GP following the injection shown in A. D: FG labeled neurons restricted to the medial part of the ipsilateral GP following the injection shown in B. Asterisks mark the boundary between the GP and $\mathrm{CPu}$. $\mathrm{CPu}$, caudate putamen; EP, entopeduncular nucleus; GP, globus pallidus; IC, internal capsule; OT, optic tract. Bar $=400 \mu \mathrm{m}$ in $A$ and $B$, and $275 \mu \mathrm{m}$ in $C$ and $D$ 
CA) was iontophoretically injected ( $5 \mu \mathrm{A}, 7 \mathrm{~s}$ on $7 \mathrm{~s}$ off, 12-20 min) into the GP of 10 adult rats via glass micropipettes with inside tip diameters of $15-30 \mu \mathrm{m}$. Ten days later the animals were perfused with $200 \mathrm{ml}$ of PBS with $0.1 \%$ sodium nitrite followed by $300-400 \mathrm{ml}$ of $4 \%$ paraformaldehyde $(n=2)$ or $4 \%$ paraformaldehyde with $0.1 \%$ glutaraldehyde $(n=8)$ in PB. The brains were postfixed for $1-2 \mathrm{~h}$ in the fixative solution and cryoprotected overnight in $20 \%$ sucrose in PB. Frozen coronal or sagittal sections $(40 \mu \mathrm{m})$ were stored in 0.02 M KPBS with $0.1 \%$ sodium azide until processed for PHA-L immunocytochemistry. The sections were washed twice before incubation in goat anti-PHA-L (Vector Labs; 1:1000 dilution) for $48 \mathrm{~h}$ at $4^{\circ} \mathrm{C}$ on the rotator. The sections were washed 3 times, incubated with biotinylated rabbit antigoat antibody (Vector Labs; 1:100 dilution) for $1 \mathrm{~h}$ at room temperture, then washed 3 times before incubation in the Vectastain Elite ABC solution (Vector Labs) for $1 \mathrm{~h}$ at room temperture on the rotator. All washes were carried out in $0.02 \mathrm{M}$ KPBS with $2 \%$ normal rabbit serum and $0.3 \%$ Triton $\mathrm{X}-100$ until the wash preceding the $\mathrm{ABC}$ incubation; after that wash all steps were carried out in plain $0.02 \mathrm{M}$ KPBS.

All FG injection sites that included the EP resulted in retrogradely labeled neurons in the ipsilateral GP. Other areas that contained retrogradely labeled neurons included the $\mathrm{CPu}$, the subthalamic nucleus (STh), the pedunculopontine tegmental nucleus and the substantia nigra (SN), all of which are known afferents of the EP [12]. Retrogradely labeled neurons and processes contained strongly fluorescent golden granules that filled the cell body and proximal processes.

The FG animals were divided into groups according to the location of the center of the injection site and the extent of spread into surrounding structures. One group consisted of animals with injections confined to the EP $(n=5)$. The second group had injections centered in the most medial part of the EP which spread into the lateral hypothalamus $(n=2)$. The third group had injections centered in the ventral EP with spread of tracer into the optic tract and the medial nucleus of the amygdala $(n=7)$. The fourth group of animals had injections centered in the dorsal EP with spread of the tracer into the reticular nucleus of the thalamus (RT) and the zona incerta $(n=2)$.

The iontophoretic sites restricted to EP demonstrated the existence of the GP-EP projection but did not provide complete information as to the topography since they covered the whole extent of the nucleus in most animals. One of these animals did provide information about the topographic organization of the projection. This injection site was almost completely confined to the lateral EP and resulted in retrogradely labeled neurons primarily in the lateral part of GP (Fig. 1A,C). In the second group the injection sites were centered in the medial half of EP and resulted in retrogradely labeled neurons primarily in the medial half of GP (Fig. 1B,D). In the third and fourth groups the injections were limited to the ventral or dorsal parts of the nucleus and resulted in retrogradely labeled neurons primarily in the ventral or dorsal areas of the GP, respectively (Fig. 2). Rostral/ caudal topographic information was provided by injections from all 4 groups of animals. Sites centered in either the rostral or caudal EP resulted in labeling of neurons primarily in the corresponding area of GP and suggested a topographic organization in this dimension as well. The large number of retrogradely labeled neurons in the GP following each injection of FG into the EP (Fig. 1C,D) suggested that this projection is quite extensive.

All PHA-L injection sites that included the GP resulted in substantial anterograde labeling in the ipsilateral EP. Many labeled fibers were noted to have en passant varicosities and terminal boutons in the EP. Other structures that also contained anterogradely labeled fibers and terminals were the CPu, the RT, the STh and

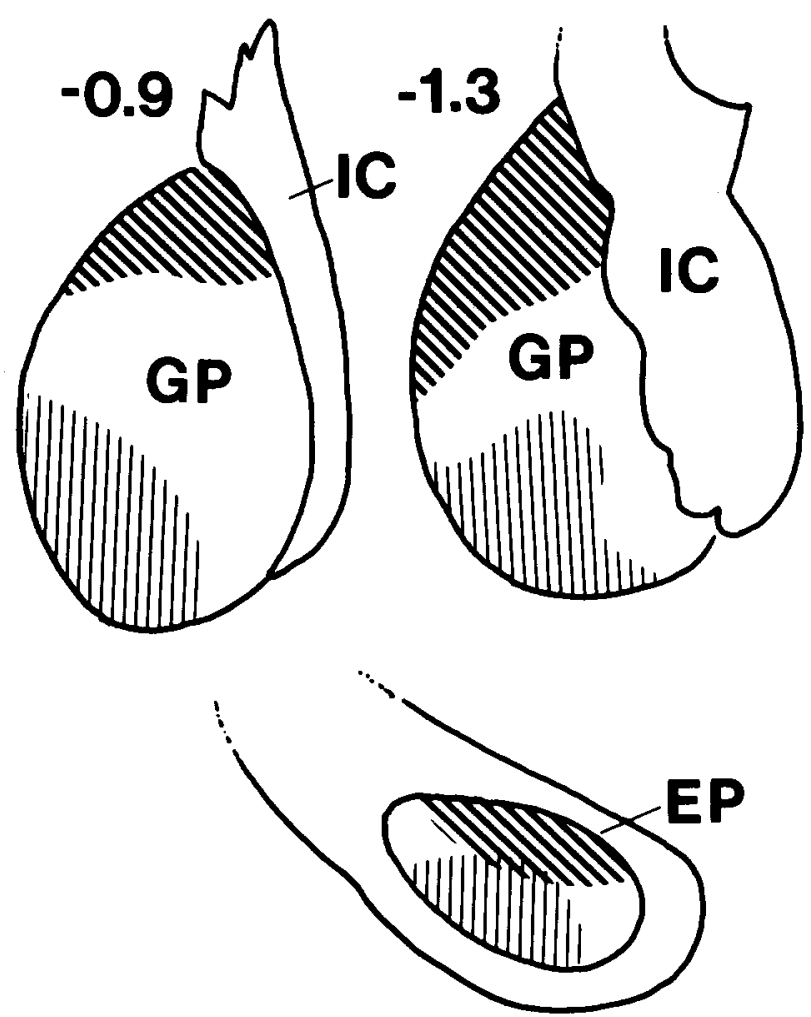

Fig. 2. Schematic representation of the dorsal/ventral topography of the GP to EP projection. FG injections from two different animals that included only the dorsal (angled lines) or ventral (vertical lines) part of EP resulted in selective transport to the respective parts of GP. The two different rostral/caudal levels of GP correspond to the -0.9 and -1.3 AP levels in the atlas of Paxinos and Watson [13]. 

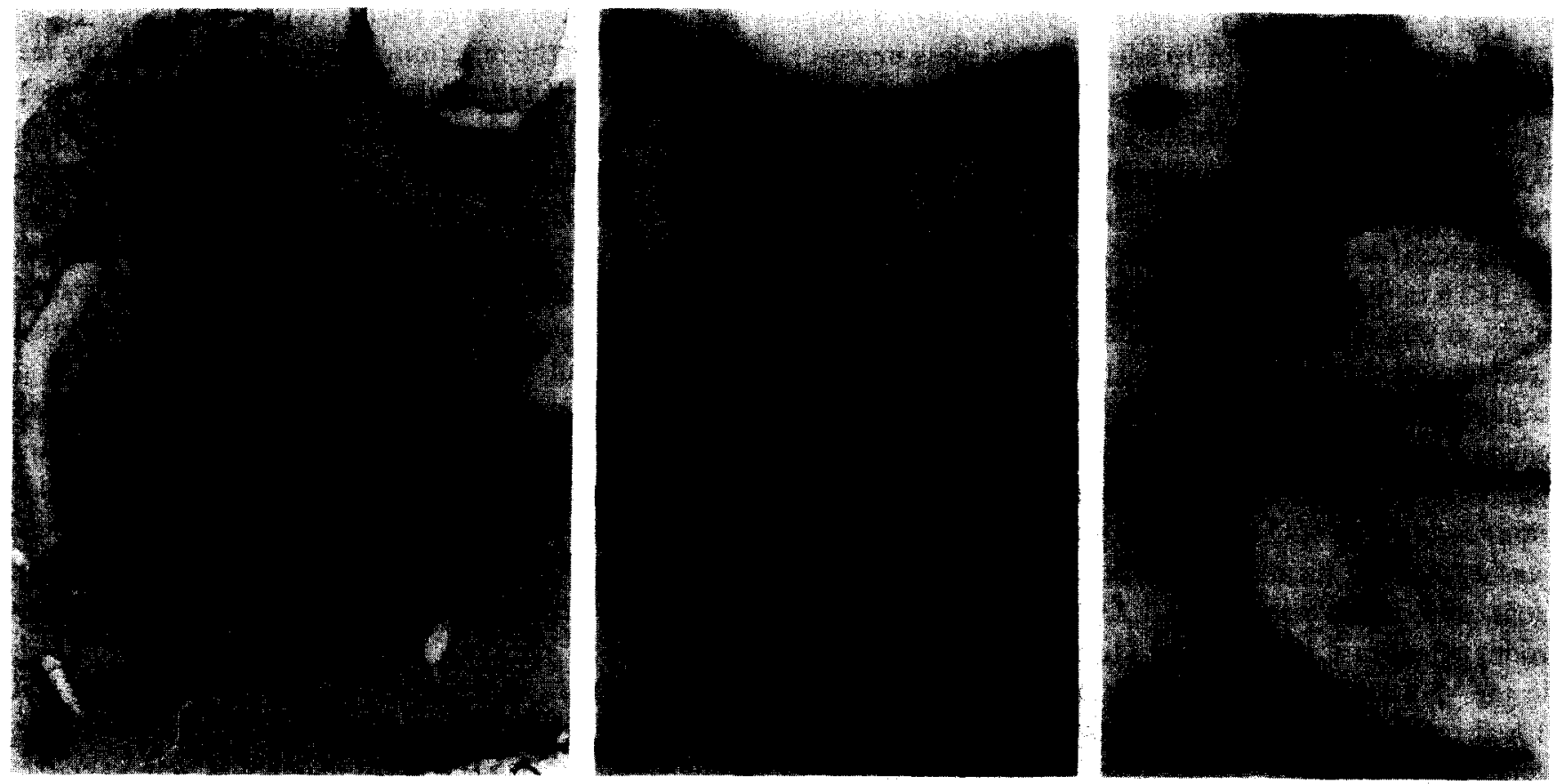

Fig. 3. A: PHA-L injection site in the GP; the pipette was angled to avoid the CPu. B and C: anterogradely labeled fibers with boutons forming apparent terminals on the proximal dendrites and somata of neurons (arrowheads) in the ipsilateral EP following the injection shown in A. B: note how the labeled fibers cover the soma of an EP neuron. Bar $=800 \mu \mathrm{m}$ in A, and $25 \mu \mathrm{m}$ in B and C.

the $\mathrm{SN}$, all previously described as projection sites of the GP [12].

Of the 10 animals that received PHA-L injections, 5 animals had injection sites confined to the GP (Fig. 3A). In two of these animals the pipette was angled to avoid the $\mathrm{CPu}$, to avoid any leakage of tracer into this known afferent of the EP. The other injection sites included surrounding structures such as the CPu laterally $(n=4)$, or the RT medially $(n=1)$.

Analysis of the injections restricted to the GP revealed dense anterograde labeling of fibers and terminals in the ipsilateral EP, even after relatively small injections (Fig. $3 \mathrm{~A}-\mathrm{C}$ ). The topography of the projection was indicated by injections that were limited to one part of the GP, and confirmed the organization suggested by the FG injections. An injection limited to the medial or lateral GP resulted in anterogradely labeled fibers primarily in the corresponding part of the ipsilateral EP.

The labeled fibers in the EP following the GP PHA-L injections were of two types. One type of fiber traveled between the fiber bundles that pierce the EP, coursing in the neuropil of the EP. These fibers had numerous varicosities, many of which surrounded the somata and the proximal dendrites of neurons in the EP (Fig. 3B,C). This pattern of fiber and terminal labeling, where the labeled fibers form a 'basket' around the somata and proximal dendrites of EP neurons, is similar to that seen in the $\mathrm{SN}$ following injections of PHA-L in the rat GP, as reported by Smith and Bolam [18]. The second type of fiber was smooth shafted with no varicosities and was restricted to the fiber bundles that penetrate the EP. This type of fiber was likely a fiber of passage, probably passing to caudal projection structures such as the STh or the SN.

Evidence presented here indicates that the GP sends a substantial, topographically organized projection to the $\mathrm{EP}$ in the rat. Although either of the tract tracing methods used in this study may provide false positive results via transport from damaged fibers of passage $[3,15,16]$, we used experimental procedures designed to minimize damage at the injection site in both cases. Our FG injections were made with a relatively dilute solution of tracer, using low currents for short periods of time. Our PHA-L injections were small, did not result in any necrosis at the injection site or abnormal retrograde or anterograde labeling that would indicate transport of the tracer by damaged fibers of passage.

Results obtained using tritiated amino acid tract tracing, which is not subject to transport by fibers of passage [4], are consistent with our findings. Carter and Fibiger [2] reported the presence of silver grains over the EP following injections of $\left[{ }^{3} \mathrm{H}\right]$ leucine into the rat GP, but could not determine if these were fibers of passage or terminals. Evidence presented here indicates that they were terminals and not fibers of passage.

Although definitive proof of this projection awaits 
study at the ultrastructural level, previous studies have shown that PHA-L labeled fibers at the light microscopic level, similar in appearance to those seen in this study, represent synaptic contacts when analyzed with an electron microscope $[18,21]$.

The existence of a direct projection from the GP to the EP would provide a route for disinhibition of EP neurons, as the GP receives an inhibitory projection from the $\mathrm{CPu}$ [5] and it has been shown that almost all GP neurons are GABAergic $[11,14]$.

The authors wish to thank Lorita Dudus for technical assistance. This work was supported by NIH NS20629 to S.W.N., NIH NS19613 to A.B.Y. and J.B.P. and the Morphology Core of P-30 Center Grant HD19258.

1 Carpenter, M.B. and Strominger, N.L., Efferent fibers of the subthalamic nucleus in the monkey, Am. J. Anat., 121 (1967) 41-72.

2 Carter, D.A. and Fibiger, H.C., The projections of the entopeduncular nucleus and globus pallidus in rat as demonstrated by autoradiography and horseradish peroxidase histochemistry, J. Comp. Neurol., 177 (1979) 113-124.

3 Cliffer, K.D. and Giesler, G.J., PHA-L can be transported anterogradely through fibers of passage, Brain Res., 458 (1988) 185-191.

4 Cowan, W.M. and Cuenod, M., The use of axonal transport for the study of neural connections: a retrospective survey. In W.M Cowan and M. Cuenod (Eds.), The Use of Axonal Transport for Studies of Neuronal Connectivity, Elsevier, New York, 1975, pp. 3-24.

5 Graybiel, A.M., Neurotransmitters and neuromodulators in the basal ganglia, Trends Neurosci., 13 (1990) 244-254.

6 Hazrati, L.-N., Parent, A., Mitchell, S. and Haber, S.N., Evidence for interconnections between the two segments of the globus pallidus in primates: a PHA-L anterograde tracing study, Brain Res., 533 (1990) 171-175.

7 Kitai, S.T. and Kita, H., Intracellular recording and labeling of globus pallidus neurons in the rat, Soc. Neurosci. Abstr., 10 (1984) 703.
8 Martinez, A., Fiber connections of the globus pallidus in man, J. Comp. Neurol., 117 (1961) 37-42.

9 Mettler, F.A., Fiber connections of the corpus striatum of the monkey and baboon, J. Comp. Neurol., 82 (1945) 169-204.

10 Nauta, W.J.H. and Mehler, W.R., Projections of the lentiform nucleus in the monkey, Brain Res., 1 (1966) 3-42.

11 Oertel, W.H., Nitsch, C. and Mugnaini, E., Immunocytochemical demonstration of the GABA-ergic neurons in rat globus pallidus and nucleus entopeduncularis and their GABA-ergic innervation. In R.G. Hassler and J.F. Christ (Eds.), Advances in Neurology, Vol. 40, Raven, New York, 1984, pp. 91-98.

12 Parent, A., Extrinsic connections of the basal ganglia, Trends Neurosci., 13 (1990) 254-258.

13 Paxinos, G. and Watson, C., The Rat Brain in Stereotaxic Coordinates, 2nd edn., Academic Press Inc., Orlando, 1986.

14 Ribak, C.E., The GABAergic neurons of the extrapyramidal system as revealed by immunocytochemistry. In G. Di Chiara and G.L. Gessa (Eds.), GABA and The Basal Ganglia, Raven, New York, 1981, pp. 23-36.

15 Schmued, L.C. and Fallon, J.H., Fluoro-Gold: a new fluorescent retrograde axonal tracer with numerous unique properties, Brain Res., 377 (1986) 147-154.

16 Schofield, B.R., Uptake of Phaseolus vulgaris leucoagglutinin by axons of passage, J. Neurosci. Methods, 35 (1990) 47-56.

17 Shu, S.Y. and Peterson, G.M., Anterograde and retrograde axonal transport of Phaseolus vulgaris leucoagglutinin from the globus pallidus to the striatum of the rat, J. Neurosci. Methods, 25 (1988) $175-180$.

18 Smith, Y. and Bolam, J.P., Neurons of the substantia nigra reticulata receive a dense GABA-containing input from the globus pallidus in the rat, Brain Res., 493 (1989) 160-167.

19 Staines, W.A., PHAL studies of the efferent connections of rat globus pallidus, Soc. Neurosci. Abstr., 14 (1988) 1028.

20 Wilson, S.A.K., An experimental research into the anatomy and physiology of the corpus striatum, Brain, 36 (1913-1914) 427-492.

21 Wouterlood, F.G. and Groenewegen, H.J., Neuroanatomical tracing by use of Phaseolus vulgaris-leucoagglutinin (PHA-L): electron microscopy of PHA-L-filled neuronal somata, dendrites, axons and axon terminals, Brain Res., 326 (1985) 188-191. 